\title{
Barreiras e estratégias para implementação de Diretrizes Nacionais do Parto Normal no Brasil
}

\author{
Jorge Otávio Maia Barreto, ${ }^{1}$ Maritsa C. Bortoli, ${ }^{2}$ Cézar D. Luquine Jr., ${ }^{2}$ Cintia F. Oliveira, ${ }^{2}$ \\ Tereza S. Toma, ${ }^{2}$ Aline A. V. Ribeiro, ${ }^{2}$ Taís R. Tesser, ${ }^{2}$ Daphne Rattner, ${ }^{3}$ Avila Vidal, ${ }^{3}$ \\ Yluska Mendes, ${ }^{3}$ Viviane Carvalho, ${ }^{3}$ Mônica Almeida Nerit e Evelina Chapman ${ }^{1}$
}

Como citar Barreto JOM, Bortoli MC, Luquine Jr. CD, Oliveira CF, Toma TS, Ribeiro AAV, et al. Barreiras e estratégias para implementação de Diretrizes Nacionais do Parto Normal no Brasil. Rev Panam Salud Publica. 2020;44:e120. https://doi.org/10.26633/ RPSP.2020.120

RESUMO

\begin{abstract}
Este relato apresenta os processos e resultados de um projeto de tradução do conhecimento desenvolvido em três etapas para identificar barreiras e estratégias para a efetiva implementação das Diretrizes Nacionais do Parto Normal no Brasil. O referencial metodológico adotado foi o da iniciativa iPIER e das ferramentas SUPPORT para Políticas Informadas por Evidências. Na primeira etapa, a qualidade das Diretrizes foi avaliada e as barreiras à implementação das recomendações foram identificadas, considerando evidências globais e análise de contribuições de uma consulta pública. Na segunda etapa, uma síntese de evidências informou um diálogo deliberativo para priorização de barreiras. Por fim, uma nova síntese de evidências informou um segundo diálogo deliberativo e apresentou seis opções para abordar as barreiras priorizadas: 1) incentivar o uso de estratégias de intervenção multifacetadas; 2) promover intervenções educativas para o uso de diretrizes em saúde; 3) realizar auditoria e feedback para adequação da prática profissional; 4) utilizar lembretes para mediar a interação entre profissionais e usuários; 5) viabilizar intervenções mediadas por pacientes e 6) incluir líderes de opinião para incentivar o uso de diretrizes em saúde. Os processos e resultados de cada etapa do projeto foram documentados e apresentados para informar a atualização das Diretrizes e o desenvolvimento de plano de implementação das recomendações. A efetiva implementação das Diretrizes é relevante para a melhoria da atenção ao parto e nascimento no Brasil.
\end{abstract}

Palavras-chave Política informada por evidências; ciência da implementação; prática clínica baseada em evidências; guia de prática clínica; parto normal; Brasil.

Em 2018, uma iniciativa conjunta entre a Organização Pan-Americana da Saúde (OPAS), a Aliança para Pesquisa em Políticas e Sistemas de Saúde (AHPSR) e o Programa Especial de Pesquisa e Treinamento em Doenças Tropicais (TDR) lançou a convocatória de projetos de pesquisa denominada "Incorporação de pesquisas para os Objetivos de Desenvolvimento Sustentável" (Embedding Research for the Sustainable Development Goals, ER-SDG) (1). A ER-SDG adotou o modelo bem-sucedido da iniciativa Improving Programme
Implementation through Embedded Research (iPIER), que apoia a integração de pesquisas na implementação de programas e políticas de saúde. Parte do processo consiste em identificar barreiras e facilitadores e incorporar esses resultados na formulação e/ou melhoria das políticas e práticas de saúde pública (2).

O projeto "Barreiras e estratégias para a implementação das Diretrizes do Parto Normal no Brasil", contemplado na convocatória ER-SDG, teve o objetivo de identificar barreiras

\footnotetext{
Fundação Oswaldo Cruz (Fiocruz), Brasília, DF, Brasil. $\bowtie$ Jorge Otávio Maia Barreto, jorge.barreto@fiocruz.br

2 Secretaria de Estado da Saúde de São Paulo, Instituto de Saúde, São Paulo (SP), Brasil.
}

\footnotetext{
Universidade de Brasília (UnB), Faculdade de Saúde, Departamento de Saúde Coletiva, Brasília (DF), Brasil.

4 Universidade Federal da Bahia (UFBa), Instituto de Saúde Coletiva, Salvador (BA), Brasil.
}

Este é um artigo de acesso aberto distribuído sob os termos da Licença Creative Commons Attribution-NonCommercial-NoDerivs 3.0 IGO, que permite o uso, distribuição e reprodução em qualquer meio, desde que o trabalho original seja devidamente citado. Não são permitidas modificações ou uso comercial dos artigos. Em qualquer reprodução do artigo, não deve haver nenhuma sugestão de que a OPAS ou o artigo avaliem qualquer organização ou produtos específicos. Não é permitido o uso do logotipo da OPAS. Este aviso deve ser preservado juntamente com o URL original do artigo. 
e estratégias para a implementação dessas Diretrizes (3) mediante aplicação de processos e ferramentas de tradução do conhecimento. As Diretrizes compreendem 225 recomendações para o cuidado à parturiente e ao recém-nascido, em oito áreas temáticas: 1) local de assistência ao parto; 2) cuidados gerais durante o trabalho de parto; 3) alívio da dor no trabalho de parto; 4) assistência no primeiro período do parto; 5) assistência no segundo período do parto; 6) assistência no terceiro período do parto; 7) cuidados maternos imediatamente após o parto; e 8) assistência ao recém-nascido.

Apesar do reconhecido potencial das diretrizes clínicas para apoiar a melhoria da organização dos serviços e desfechos de saúde, esses documentos ainda são subutilizados (4-6), uma vez que sua implementação geralmente não é planejada, ou tem pouca eficiência (7). No caso da assistência ao parto no Brasil, por exemplo, apesar dos avanços conquistados nas últimas décadas, o modelo obstétrico ainda é fortemente medicalizado e marcado por práticas desnecessárias e potencialmente iatrogênicas, como as cesarianas eletivas, além de frequentes relatos de violência obstétrica $(8,9)$, fazendo com que a implementação das recomendações seja ainda mais desafiadora.

O objetivo deste relato é apresentar processos e resultados do Projeto Barreiras e estratégias para a implementação das Diretrizes do Parto Normal no Brasil, com ênfase nos métodos adotados e em seus produtos. Pretende-se contribuir para o aprimoramento da assistência ao parto no Brasil, bem como incentivar o uso de ferramentas de tradução do conhecimento.

\section{ETAPAS DE DESENVOLVIMENTO DO PROJETO E PRODUTOS}

O Projeto foi implementado de dezembro de 2018 a novembro de 2019 pela Fundação Oswaldo Cruz (Fiocruz Brasília), em colaboração com o Ministério da Saúde (Coordenação de Saúde das Mulheres), a Universidade de Brasília (Departamento de Saúde Coletiva) e o Instituto de Saúde da Secretaria de Estado da Saúde de São Paulo (Núcleo de Evidências em Saúde), com aprovação pelos comitês de ética da Fiocruz Brasília (CAAE 01857418.1.0000.8027) e da OPAS (PAHO-ERCS). Os participantes dos diálogos deliberativos forneceram seu consentimento informado e medidas foram adotadas para resguardar o anonimato dos dados coletados.

O desenvolvimento do Projeto seguiu um protocolo prévio (Material Suplementar, página 1) em três etapas, de acordo com seus objetivos específicos: 1) caracterização das Diretrizes do Parto Normal e do seu cenário de implementação nas maternidades do Sistema Único de Saúde (SUS); 2) identificação e priorização de barreiras e facilitadores à implementação; e 3) identificação e priorização de estratégias de implementação. A figura 1 sistematiza as etapas do Projeto.

FIGURA 1. Fluxograma das etapas, atividades e produtos do projeto “Barreiras e estratégias para a implementação das Diretrizes do Parto Normal no Brasil"”

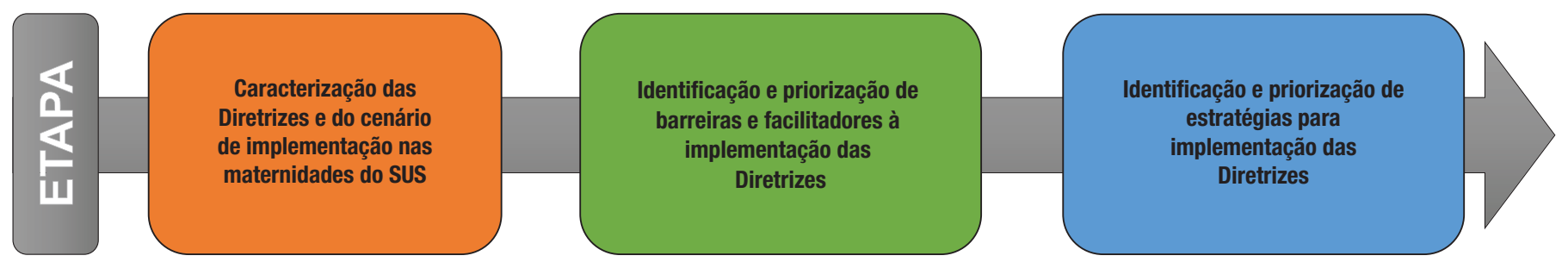

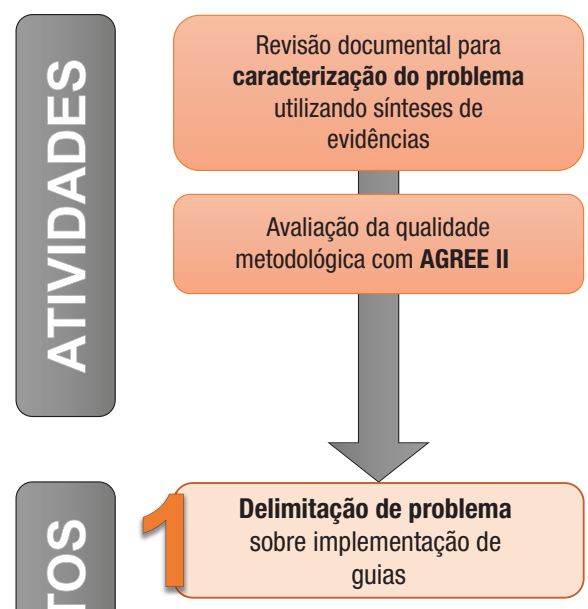
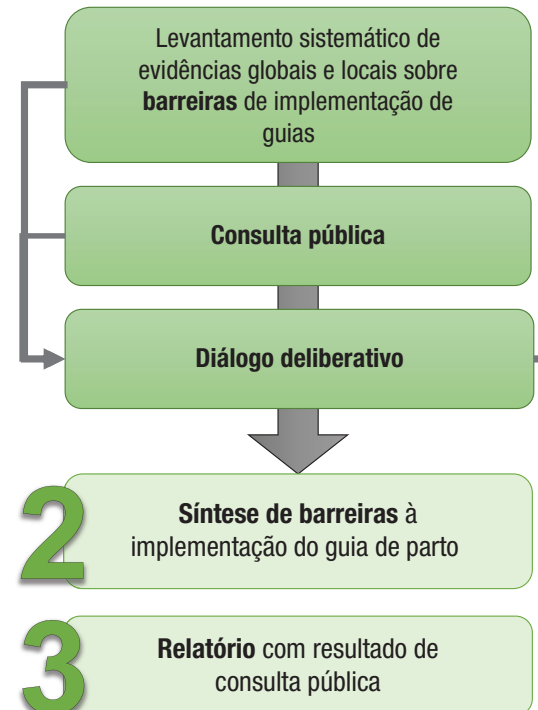

Relatório com resultado de consulta pública

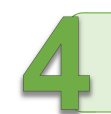

Relatório do diálogo deliberativo 


\section{Etapa 1: Caracterização das Diretrizes do Parto Normal e do cenário de implementação nas maternidades do SUS}

Uma revisão documental foi conduzida de outubro de 2018 a março de 2019 para caracterizar o processo de elaboração das Diretrizes e seu cenário de implementação, a fim de fundamentar o protocolo do Projeto (Material Suplementar, página 1). Além disso, uma avaliação da qualidade metodológica das Diretrizes foi realizada, usando o instrumento AGREE II (10). Os resultados foram comparados aos parâmetros da ponderação proposta pelo AGREE II (Material Suplementar, página 10).

A avaliação identificou duas fragilidades nas Diretrizes: a representatividade de alguns atores interessados ficou aquém do desejado, principalmente das diferentes categorias profissionais e de usuários, ainda que o processo tenha sido participativo; e a ausência de um plano de implementação e monitoramento de processos influiu na avaliação quanto à sua aplicabilidade. Dessa forma, os avaliadores recomendaram a atualização das Diretrizes, com ajustes metodológicos e a inclusão de um plano de implementação.

\section{Etapa 2: Identificação e priorização de barreiras e facilitadores à implementação}

O mapeamento de potenciais barreiras à implementação foi realizado de março a junho de 2019 em três atividades: 1) elaboração de uma síntese de evidências secundárias globais e locais, 2) análise textual das contribuições de uma consulta pública e 3) realização de um diálogo deliberativo para priorizar barreiras à implementação.

A síntese de evidências sobre barreiras à implementação de guias de prática clínica fundamentou-se nas etapas propostas pelas ferramentas SUPPORT (Supporting Policy relevant Reviews and Trials) para Políticas Informadas por Evidências (11), a partir das falhas de implementação abordadas na Etapa 1. As buscas foram conduzidas em bases eletrônicas (PubMed, LILACS, BDENF, Health Systems Evidence, Health Evidence e Epistemonikos) em março e abril de 2019, utilizando estratégias estruturadas (Material Suplementar, página 19). Foram considerados estudos secundários (revisões sistemáticas e sínteses qualitativas) e diretrizes clínicas publicados em português, inglês ou espanhol que abordassem barreiras à implementação.

Como resultado geral, 23 textos (21 artigos e dois guias clínicos) foram incluídos na síntese, extraindo-se informações sobre as barreiras à implementação, as quais foram estratificadas por nível de organização no sistema de saúde (usuários, trabalhadores, serviços e sistemas de saúde). Foi realizada a avaliação da qualidade metodológica apenas das revisões sistemáticas, usando-se a ferramenta AMSTAR (Assessing the Methodological Quality of Systematic Reviews) (12).

Na síntese dos achados, inicialmente as barreiras foram categorizadas conforme as oito seções das Diretrizes e por nível organizacional. Em seguida, as barreiras foram agrupadas por similaridade em categorias temáticas, dentro de cada nível, e finalmente reagrupadas em núcleos de sentido. Os resultados foram sistematizados em um documento (Material Suplementar, página 12), que informou o Diálogo Deliberativo de priorização de barreiras.

As barreiras identificadas relacionaram-se a 25 das 225 recomendações das Diretrizes de Parto Normal (figura 2). A síntese de evidências sobre barreiras à implementação das Diretrizes (13) é apresentado em outro artigo desta edição especial sobre os projetos da iniciativa ER-SDG.

A identificação das barreiras à implementação foi complementada pela análise dos dados textuais da "Consulta Pública de Diretrizes Nacionais de Assistência ao Parto Normal", realizada em 2016, pelo Ministério da Saúde. A consulta pública integra o processo de incorporação de tecnologias e de protocolos clínicos e diretrizes terapêuticas da Comissão Nacional de Incorporação de Tecnologias no SUS (CONITEC) e contou com 396 contribuições individuais de pessoas e instituições de vários estados do Brasil. As análises estatísticas do corpus textual da consulta pública foram realizadas com apoio do programa IRAMUTEQ (14).

$\mathrm{Na}$ análise das contribuições individuais, 93 registros foram excluídos, pois se limitavam a "sim" ou "não". As contribuições incluídas foram organizadas em um único corpus, submetido a uma análise de classificação hierárquica descendente (CHD) (14). Dessa análise inicial foram extraídos dois subcorpora, um com palavras relacionadas às barreiras e outro com palavras relacionadas aos facilitadores para a implementação das Diretrizes. Uma nova análise CHD foi realizada nesses subcorpora, para extrair trechos representativos das principais barreiras e facilitadores identificados. A sistematização dessa análise da consulta pública é apresentada na figura 3. Um relatório detalhado pode ser encontrado no Material Suplementar, página 69).

Para priorizar as barreiras identificadas pela síntese de evidências e pela análise das contribuições da consulta pública, foi realizado um diálogo deliberativo com nove participantes, entre gestoras da Coordenação de Saúde da Mulher do Ministério da Saúde e pesquisadoras de temas relativos ao assunto do diálogo. A organização e a realização seguiram as ferramentas SUPPORT (15). As participantes receberam previamente os dois relatórios sobre barreiras de implementação e o diálogo deliberativo seguiu a regra de Chatham House, que autoriza os participantes a comentarem externamente as discussões da reunião, porém sem atribuir autoria às falas.

Após apresentação das mensagens-chave, foi conduzida uma discussão estruturada e três barreiras à implementação foram consideradas prioritárias: 1) o modelo de atenção centrado no médico, dificultando a participação das parturientes e de outros profissionais na assistência ao parto; 2 ) o modelo de formação profissional, que reproduz práticas sem respaldo de evidências científicas e possui orientação biomédica em detrimento de aspectos humanistas do cuidado, dificultando a incorporação de novas evidências às práticas assistenciais; e 3) a insuficiência e inadequação da infraestrutura nos serviços de saúde para apoiar a implementação das melhores estratégias de cuidado. As deliberações realizadas foram reportadas em um relatório sintético (Material Suplementar, página 75).

\section{Etapa 3: Identificação e priorização de estratégias de implementação}

A etapa 3 foi realizada de julho a outubro de 2019. A partir dos resultados do diálogo deliberativo, outra síntese de evidências abordou opções para apoiar a implementação das Diretrizes, considerando as barreiras de implementação priorizadas. As opções foram identificadas a partir de buscas sistemáticas, realizadas em julho de 2019, em seis bases de 
FIGURA 2. Relações entre as barreiras à implementação apresentadas na síntese de evidências globais e as Diretrizes Nacionais de Assistência ao Parto Normal

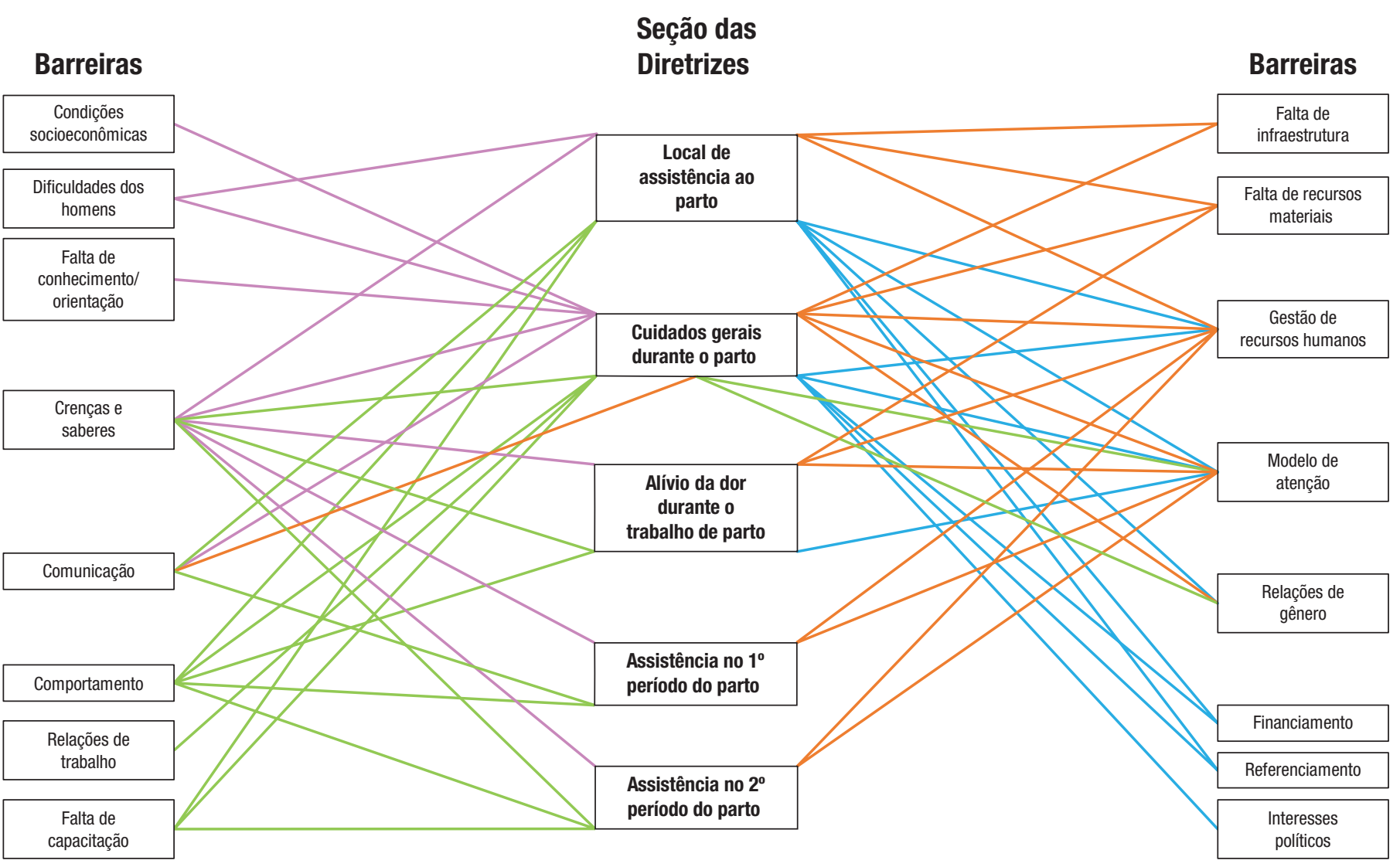

Nível da organização

\begin{tabular}{|l|l|l|l|}
\hline USUÁRIOS & TRABALHADORES & SERVIÇOS DE SAÚDE & SISTEMA DE SAÚDE \\
\hline
\end{tabular}

dados (Scopus, PubMed, BVS-LILACS, Epistemonikos, Health Systems Evidence e Health Evidence) (Material Suplementar, página 79). Foram incluídas revisões sistemáticas publicadas em inglês, português ou espanhol, que abordassem estratégias para implementação de diretrizes clínicas em serviços e/ou sistemas de saúde. A seleção foi realizada por meio da plataforma Rayyan QCRI (16) e a qualidade metodológica das revisões incluídas foi avaliada com a ferramenta AMSTAR (12).

A busca recuperou 3830 documentos, dos quais 3780 foram excluídos por serem duplicados (585) ou por não atenderem aos critérios de inclusão (3 195); 50 artigos foram selecionados para leitura completa, sendo 29 incluídos. Os dados foram extraídos e categorizados conforme a taxonomia para intervenções em sistemas de saúde do Cochrane Effective Practice and Organisation of Care Group (EPOC) (17). A síntese de evidências produziu seis opções e subsidiou um segundo diálogo deliberativo, com o objetivo de contribuir para a formulação de um plano de implementação das Diretrizes.

É importante ressaltar que nem todas as opções identificadas apresentam o mesmo nível de confiança sobre sua efetividade, nem são condicionadas pelos mesmos fatores de implementação. Além disso, as opções incluem desde ações isoladas até intervenções multifacetadas, exigindo uma análise sobre potenciais benefícios, riscos e incertezas. As opções foram organizadas considerando a qualidade metodológica das revisões utilizadas em sua composição. A seguir é apresentada uma breve descrição das seis opções. A versão completa da síntese de evidências pode ser encontrada no Material Suplementar (página 79).

A opção 1, incentivar o uso de estratégias de intervenção multifacetadas, envolve a combinação de duas ou mais intervenções. Entre as 19 revisões sistemáticas incluídas nessa opção (18-36), cinco foram consideradas de alta qualidade metodológica, nove de média qualidade e cinco de baixa qualidade. Os estudos analisaram uma gama muito ampla de combinações de intervenções, por exemplo, reuniões educacionais administradas por líderes de opinião e visitas de sensibilização educacional (18); ou envio de materiais por correio, realização de telefonemas de acompanhamento e auditoria posterior (19). A eficácia de intervenções multifacetadas no campo da obstetrícia depende da natureza das estratégias usadas e os seus resultados são diferentes de acordo com o comportamento visado, a unidade de alocação e os prestadores de serviço (20). Assim, a escolha adaptada das intervenções que vão compor uma estratégia multifacetada é determinante para a eficácia desta opção.

A opção 2, promover intervenções educativas para o uso de diretrizes em saúde, foi informada por 18 revisões sistemáticas 
FIGURA 3. Principais barreiras à implementação das Diretrizes Nacionais de Assistência ao Parto Normal identificadas na análise do corpus da consulta pública

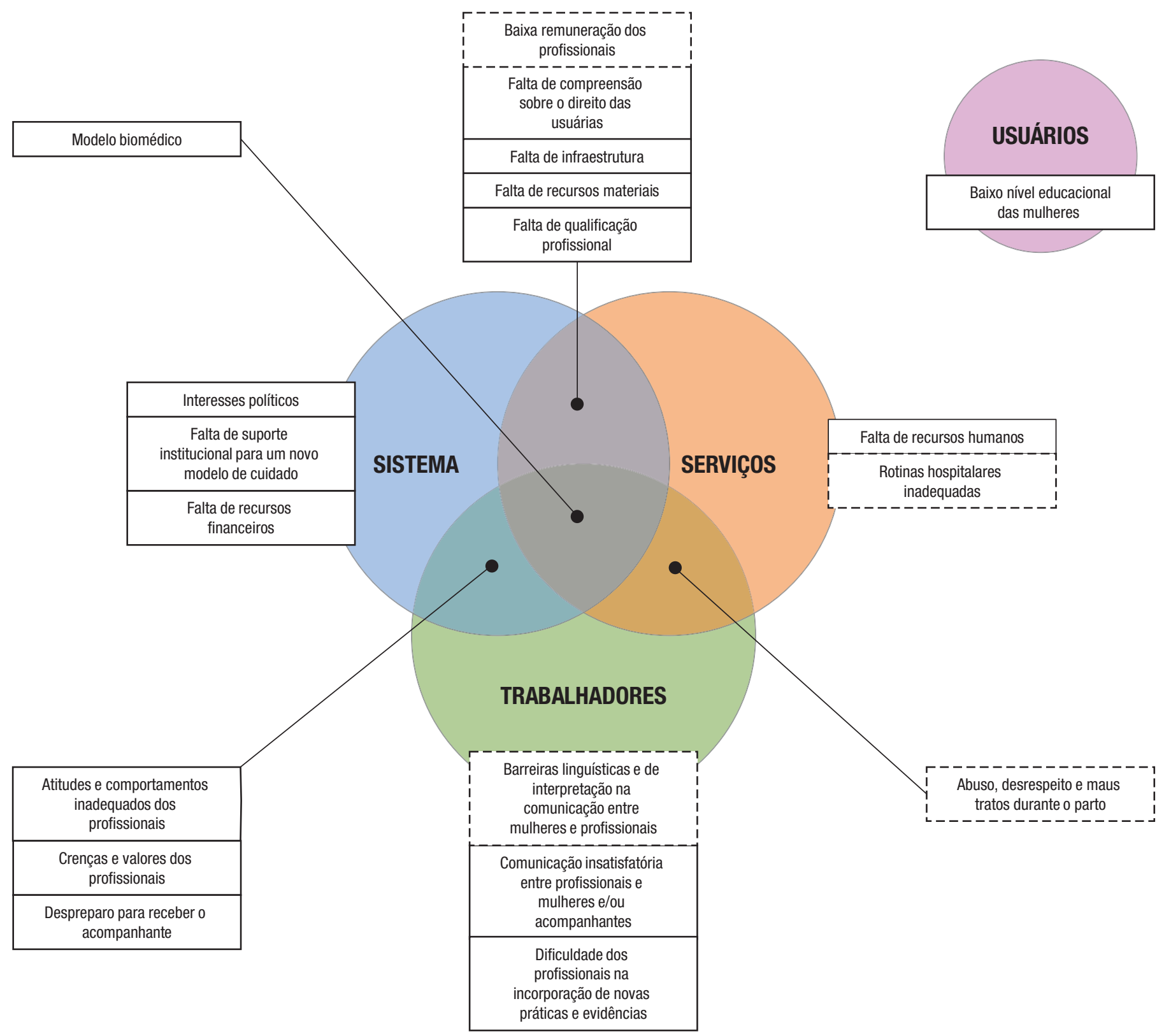

(19-21, 23-27, 29, 34, 37-39), sendo cinco de alta qualidade metodológica, oito de média qualidade e cinco de baixa qualidade. As intervenções foram agrupadas em três categorias: materiais educativos $(19,21,26,34,37)$, encontros educativos $(19,23,25-27,29,37)$ e visitas de divulgação educacional (educational outreach visits) ou detalhamento acadêmico $(19,20,24$, $26,34-39)$. As intervenções com materiais educativos se referem à distribuição de informações a indivíduos ou grupos para apoiar o atendimento clínico, ou seja, qualquer intervenção na qual se "entregam" informações. Os encontros educativos são definidos como cursos, oficinas, conferências ou outras reuniões educacionais. As visitas de divulgação educativa ou detalhamento acadêmico são visitas realizadas por uma pessoa treinada a profissionais de saúde em seus próprios locais de trabalho, para fornecer informações com o objetivo de modificar práticas (17).

A opção 3, realizar auditoria e feedback para adequação da prática profissional, envolve ações de devolutiva aos profissionais sobre seu desempenho para encorajá-los a seguirem um padrão de referência (40). Conforme 10 revisões sistemáticas $(19,20,24$, $26,34,36,37,40-42)$, sendo uma de alta qualidade metodológica, cinco de média qualidade e quatro de baixa qualidade, as intervenções de auditoria e feedback são utilizadas comumente em serviços de saúde para melhorar o desempenho dos profissionais. Uma síntese da performance dos profissionais em um determinado período de tempo é apresentada em formatos 
variados (40), como relato verbal, impresso ou por meio eletrônico, e pode incluir sugestões específicas para melhoria da prática clínica (43). Entre as especificidades dessa opção, vale notar a melhor aceitação por enfermeiras na prática obstétrica do que em outros contextos clínicos (20).

A opção 4, utilizar lembretes para mediar a interação entre profissionais e usuários, também foi informada por 10 revisões sistemáticas $(14,19,20,22,26,35-37,44,45)$, sendo uma de alta qualidade metodológica, cinco de média qualidade e quatro de baixa qualidade. Essa opção envolve o uso de lembretes, que, entre os sistemas de apoio à decisão dos profissionais de saúde, são amplamente utilizados com o objetivo de estimular ou auxiliar a memória (44). Lembretes são caracterizados como ações para mediar o comportamento de profissionais de saúde durante a interação com os usuários (17). Essas intervenções podem auxiliar os profissionais de saúde a superarem problemas, como atos de omissão e falta de acesso ou sobrecarga de informações. Os lembretes são capazes de estimular a lembrança de informações que os profissionais já sabem ou se esperaria que soubessem e fornecem orientações em formato acessível e relevante num momento particularmente conveniente (44). A estrutura dos lembretes pode variar em conteúdo (sugestão de exames ou referência à literatura, entre outros), formato (impresso, eletrônico, gerado por computador e entregue em papel, entre outros) e organização. Além disso, podem ser compartilhados com os profissionais pela tela do computador, por e-mail ou pelo prontuário dos pacientes $(44,45)$.

A opção 5, viabilizar intervenções mediadas por pacientes, baseou-se em cinco revisões sistemáticas (19, 24, 30, 37, 38), sendo uma de alta qualidade metodológica, três de média qualidade e uma de baixa qualidade. Está relacionada a qualquer intervenção destinada a alterar o desempenho dos profissionais de saúde por meio de interações com o paciente ou ações que envolvem repassar ou receber informações para/ do paciente (43), como quando o paciente comunica aos profissionais informações sobre sua saúde; quando o paciente recebe informações sobre sua saúde (por exemplo, sobre cuidados recomendados); educação de pacientes (por exemplo, sobre a sua condição e opções de cuidado); e interações para apoiar a tomada de decisão por parte do paciente, em que ele recebe informações sobre opções de tratamento, incluindo riscos e benefícios (27).

A opção 6, envolver líderes de opinião para incentivar o uso de diretrizes em saúde, baseou-se em cinco revisões sistemáticas $(19,20,26,37,46)$, sendo três de média qualidade metodológica e duas de baixa qualidade. Os líderes de opinião locais são prestadores de cuidados de saúde ou especialistas em uma determinada área que oferecem apoio permanente para o uso de diretrizes e das melhores práticas baseadas em evidências (37). Esse tipo de estratégia parte do pressuposto de que é possível gerar mudanças por meio de interação e da influência de pessoas legitimadas por seu conhecimento (20). A identificação e atuação de líderes de opinião locais podem promover a boa prática clínica (17). Esses líderes podem ser, por exemplo, clínicos reconhecidos pelos colegas como profissionais respeitados e eficazes na comunicação de informações (20), profissionais de saúde reconhecidos em sua comunidade (19) ou mesmo peritos em determinada área que prestam apoio contínuo a outros profissionais, ajudando-os a melhorar sua prática com base em evidências científicas (37).

\section{Diálogo deliberativo sobre estratégias de implementação}

A síntese de evidências sobre estratégias de implementação informou um segundo diálogo deliberativo, orientado pelas ferramentas SUPPORT (15). Participaram 15 atores estrategicamente selecionados para representar os grupos e níveis interessados no tema, entre gestores, pesquisadores, profissionais de saúde e representantes de usuários. O diálogo promoveu a interação mediada para deliberações sobre a importância de combinar intervenções de forma a alcançar os melhores resultados na implementação de diretrizes e de intensificar esse debate no âmbito da formação de profissionais de saúde. Da mesma forma, ressaltou-se a necessidade de fortalecer a participação ativa das mulheres e de seus acompanhantes, tanto na formulação de políticas quanto nos momentos de decisão acerca do parto de seus próprios filhos.

Os participantes destacaram a necessidade de atualizar periodicamente as Diretrizes, reforçando a necessidade de participação das mulheres e a representatividade dos demais atores sociais interessados, além de garantir o uso sistemático de evidências científicas de qualidade. Todavia, entendeu-se também que a efetiva utilização dessas Diretrizes nos serviços e na prática individual dos trabalhadores só ocorrerá a partir do comprometimento de gestores locais com a mudança do modelo assistencial. Além disso, sugeriu-se informar com mais clareza o público específico a quem se destinam as recomendações propostas nas Diretrizes (usuários, profissionais e/ou gestores), inclusive com a produção de versões adaptadas em linguagem acessível, por exemplo, para as mulheres atendidas pelos serviços de saúde.

Para alcançar esses objetivos, o grupo participante do diálogo propôs a elaboração de um plano de implementação para as Diretrizes, composto de estratégias explícitas e fundamentadas, com o objetivo de melhorar a adesão às recomendações de forma consistente pelos profissionais nos serviços de saúde. $\mathrm{O}$ relatório do segundo diálogo deliberativo pode ser encontrado no Material Suplementar (página 111).

\section{DISCUSSÃO}

A convocatória ER-SDG teve o objetivo de integrar resultados de pesquisa à implementação de programas e políticas de saúde (1). Como parte dessa iniciativa, o projeto relatado neste artigo adotou o paradigma da tradução integrada do conhecimento (47) e gerou processos e produtos para apoiar o uso sistemático e transparente do conhecimento científico na tomada de decisão, notadamente as sínteses de evidências e os diálogos deliberativos, reconhecidos como ferramentas efetivas de tradução do conhecimento (48-50).

O projeto abordou o desafio da implementação, inclusive as fragilidades no processo de elaboração das Diretrizes somadas a barreiras em diferentes níveis, do individual ao sistêmico. Diante disso, é mais ainda fundamental que as estratégias voltadas à problemática abordada neste relato sejam informadas por evidências científicas, a fim de consubstanciar um plano efetivo de implementação.

As duas sínteses de evidências destacam-se como produtos de tradução do conhecimento, apresentando de forma acessível e sistemática um conjunto de informações sobre barreiras e estratégias de implementação a serem consideradas pelos 
tomadores de decisão. Da mesma forma, os diálogos deliberativos mostraram que podem agregar informações valiosas sobre as visões, experiências e opiniões de legítimos interessados no enfrentamento do problema abordado, corroborando seu uso como ferramenta para integração entre evidências científicas (globais) e coloquiais (locais) (47).

Algumas limitações sobre os documentos apresentados neste relato merecem ser explicitadas. A primeira síntese de evidências identificou barreiras à implementação relacionadas com 25 das 225 recomendações que integram as Diretrizes. Esta importante lacuna pode estar relacionada com a inclusão de estudos secundários apenas. Embora a escolha metodológica tenha sido adequada ao desenvolvimento do Projeto, é possível que informações importantes não tenham sido consideradas. Já na síntese sobre opções para apoiar a implementação das Diretrizes, constatou-se a escassez de revisões sistemáticas sobre intervenções para diretrizes obstétricas. Por isso, evidências indiretas sobre a implementação de diretrizes em geral foram usadas. Essas limitações devem ser consideradas para equilibrar os julgamentos sobre a aplicabilidade dos resultados descritos, em especial das opções de política, ao contexto do sistema de saúde brasileiro.

A limitação temporal do desenvolvimento do projeto relatado, por sua vez, impôs influxo dessa constrição para o desenvolvimento dos processos e produtos. No entanto, o protocolo de pesquisa foi integralmente implementado; ao passo que é preciso reconhecer que, por sua vez, o processo de tomada de decisão enfrenta, comumente, a restrição de recursos imateriais, em especial temporais, mas também logísticos e cognitivos (51).

Por fim, é recomendável a atualização das recomendações das Diretrizes e a inclusão de um plano de implementação, priorizando a participação de mulheres nesse processo e delimitando objetivamente os destinatários de cada recomendação (usuários, profissionais de saúde ou gestores dos serviços e do sistema). A implementação efetiva das Diretrizes requer mudanças para a superação do modelo de assistência ao parto vigente no SUS, sendo necessário também fortalecer outras estratégias em curso no Brasil, para promover a fisiologia do nascimento, a incorporação de práticas seguras e baseadas em evidências e a garantia dos direitos sexuais e reprodutivos.

Contribuição dos autores. JOMB, EC e MAN conceberam o projeto e coordenaram sua execução. MCB, CDLJ, CFO, TST, AAVR, TRT, DR, AV, YM e VC estiveram envolvidos em diferentes etapas da execução do Projeto e desenvolvimento dos resultados apresentados. JOMB redigiu a primeira versão do manuscrito. Todos os autores contribuíram, revisaram e aprovaram a versão final do manuscrito.

Agradecimentos. Registramos nosso agradecimento aos colegas que colaboraram com a avaliação AGREE II das Diretrizes Nacionais de Assistência ao Parto Normal no Brasil, Airton Stein (Universidade Federal de Ciências da Saúde de Porto Alegre) e Daniela Melo (Universidade Federal de São Paulo). Agradecemos também a Mônica Neri e Gisele Bortolini pelo suporte institucional indispensável ao desenvolvimento do Projeto enquanto estiveram à frente da Coordenação de Saúde das Mulheres, no Ministério da Saúde.

\section{Conflitos de interesse. Nada declarado pelos autores.}

Financiamento. Este estudo foi financiado pela Organização Pan-Americana da Saúde (OPAS/OMS), pelo Programa Especial de Pesquisa e Treinamento em Doenças Tropicais (TDR) e pela Aliança para Pesquisa em Políticas e Sistemas de Saúde (AHPSR) através de uma doação ao Projeto "Barreiras e estratégias para a implementação das Diretrizes do Parto Normal no Brasil [Chamada para propostas Incorporação de pesquisas para os Objetivos de Desenvolvimento Sustentável (ER-SDG)]". Os financiadores não tiveram nenhum papel no desenho, análise, interpretação dos dados e redação do manuscrito.

Declaração. As opiniões expressas no manuscrito são de responsabilidade exclusiva dos autores e não refletem necessariamente a opinião ou política da RPSP/PAJPH ou da Organização Pan-Americana da Saúde (OPAS).

\section{REFERÊNCIAS}

1. Organización Panamericana de la Salud - OPAS. Convocatoria: Incorporación de la investigación para avanzar en el cumplimiento de los Objetivos de Desarrollo Sostenible (ER-SDG). 2018. Disponível em: https://www.paho.org/hq/index.php?option $=$ com_content $=$ article $=12040$ : call-for-applications-embedding-research-for-the-sustainable-development-goals $=41749=\mathrm{es}$ Acessado em 17 de maio de 2020.

2. Langlois E V, Mancuso A, Elias V, Reveiz L. Embedding implementation research to enhance health policy and systems: a multi-country analysis from ten settings in Latin America and the Caribbean. Health Res Policy Sys. 2019;17:85. https://doi.org/10.1186/s12961019-0484-4

3. Brasil. Ministério da Saúde. Diretrizes nacionais de assistência ao parto normal: versão resumida. Brasília: Ministério da Saúde; 2017. Disponível em: https://pesquisa.bvsalud.org/bvsms/resource/ pt/mis-39026 Acessado em 2 de junho de 2020.

4. FitzGerald JM, Boulet L-P, McIvor RA, Zimmerman S, Chapman KR. Asthma Control in Canada Remains Suboptimal: The Reality of Asthma Control (TRAC) Study. Can Respir J. 2006;13(5):253-9. https://doi.org/10.1155/2006/753083
5. Brown LC, Johnson JA, Majumdar SR, Tsuyuki RT, McAlister FA. Evidence of suboptimal management of cardiovascular risk in patients with type 2 diabetes mellitus and symptomatic atherosclerosis. CMAJ. 2004;171(10):1189-92. https://doi.org/10.1503/ cmaj.1031965

6. Latosinsky S, Fradette K, Lix L, Hildebrand K, Turner D. Canadian breast cancer guidelines: have they made a difference? CMAJ. 2007;176(6):771-6. https://doi.org/10.1503/cmaj.060854

7. Gagliardi AR, Brouwers MC, Palda VA, Lemieux-Charles L, Grimshaw JM. How can we improve guideline use? A conceptual framework of implementability. Implementation Sci. 2011;6(1):26. https: / / doi.org/10.1186/1748-5908-6-26

8. World Health Organization (WHO). Maternal mortality in 2005: estimates developed by WHO, UNICEF, UNFPA, and the World Bank. Genebra: World Health Organization (WHO); 2007.

9. Fundação Perseu Abramo. Mulheres brasileiras e gênero nos espaços público e privado. São Paulo, SP; 2010. Disponível em: https: / / fpabramo.org.br/publicacoes/wp-content/uploads / sites/5/2017/05/pesquisaintegra_0.pdf Acessado em 10 de junho de 2020 . 
10. Brouwers MC, Kho ME, Browman GP, Burgers JS, Cluzeau F, Feder $\mathrm{G}$, et al. AGREE II: advancing guideline development, reporting and evaluation in health care. CMAJ. 2010;182(18):E839-42. https://doi. org /10.1503/cmaj.090449

11. Lavis JN, Oxman AD, Lewin S, Fretheim A. SUPPORT Tools for evidence-informed health Policymaking (STP). Health Res Policy Sys. 2009;7(S1):I1. https:/ / doi.org/10.1186/1478-4505-7-S1-I1

12. Shea BJ, Grimshaw JM, Wells GA, Boers M, Andersson N, Hamel C, et al. Development of AMSTAR: a measurement tool to assess the methodological quality of systematic reviews. BMC Med Res Methodol. 2007;7(1):10. https://doi.org/10.1186/1471-2288-7-10

13. Oliveira CF, Ribeiro AAV, Luquine Jr CD, de Bortoli MC, Toma TS, Chapman EMG, et al. Barreiras à implementação de recomendações para assistência ao parto normal: revisão rápida de evidências. Rev Panam Salud Publica. 2020;44:e132. https:/ /doi.org/10.26633/ RPSP.2020.132

14. Camargo BV, Justo AM. IRAMUTEQ: Um software gratuito para análise de dados textuais. Temas Psicol. 2013;21(2):513-8. https:// doi.org/10.9788/TP2013.2-16

15. Lavis JN, Boyko JA, Oxman AD, Lewin S, Fretheim A. SUPPORT Tools for evidence-informed health Policymaking (STP) 14: Organising and using policy dialogues to support evidence-informed policymaking. Health Res Policy Sys. 2009;7(S1):S14. https://doi. org /10.1186/1478-4505-7-S1-S14

16. Ouzzani M, Hammady H, Fedorowicz Z, Elmagarmid A. Rayyan-a web and mobile app for systematic reviews. Syst Rev. 2016;5:210. https://doi.org/10.1186/s13643-016-0384-4

17. Effective Practice and Organisation of Care (EPOC). EPOC Taxonomy. 2015. Disponível em: epoc.cochrane.org/epoc-taxonomy Acessado em 4 de junho de 2020.

18. van der Wees PJ, Jamtvedt G, Rebbeck T, de Bie RA, Dekker J, Hendriks EJM. Multifaceted strategies may increase implementation of physiotherapy clinical guidelines: a systematic review. Aust J Physiother. 2008;54(4):233-41. https://doi.org/10.1016/ S0004-9514(08)70002-3

19. Davis DA, Taylor-Vaisey A. Translating guidelines into practice: a systematic review of theoretic concepts, practical experience and research evidence in the adoption of clinical practice guidelines. CMAJ. 1997;157(4):408-16.

20. Chaillet N, Dubé E, Dugas M, Audibert F, Tourigny C, Fraser WD, et al. Evidence-based strategies for implementing guidelines in obstetrics: a systematic review. Obstet Gynecol. 2006;108(5):1234-45. https://doi.org/10.1097/01.AOG.0000236434.74160.8b

21. Ament SMC, de Groot JJA, Maessen JMC, Dirksen CD, van der Weijden T, Kleijnen J. Sustainability of professionals' adherence to clinical practice guidelines in medical care: a systematic review. BMJ Open. 2015;5(12):e008073. https://doi.org/10.1136/ bmjopen-2015-008073

22. Grimshaw J, Eccles M, Thomas R, MacLennan G, Ramsay C, Fraser C, et al. Toward evidence-based quality improvement: evidence (and its limitations) on theeffectiveness of guideline dissemination and implementation strategies 1966-1998.J Gen Intern Med. 2006;21(S2):S14-20. http://doi.org/10.1111/j.1525-1497.2006.00357.x

23. Flodgren G, Hall AM, Goulding L, Eccles MP, Grimshaw JM, Leng GC, et al. Tools2 developed and disseminated by guideline producers to promote the uptake of their guidelines. Cochrane Database Syst Rev. 2016;(8). http://doi.org/10.1002/14651858.CD010669. pub2

24. Grimshaw JM, Thomas RE, MacLennan G, Fraser C, Ramsay CR, Vale L, et al. Effectiveness and efficiency of guideline dissemination and implementation strategies. Health Technol Assess. 2004;8(6). https://doi.org/10.3310/hta8060

25. Imamura M, Kanguru L, Penfold S, Stokes T, Camosso-Stefinovic J, Shaw B, et al. A systematic review of implementation strategies to deliver guidelines on obstetric care practice in low- and middle-income countries. Int J Gynecol Obstet. 2017;136(1):19-28. http:/ / doi. org/10.1002/ijgo.12005

26. Dijkstra R, Wensing $M$, Thomas R, Akkermans R, Braspenning J, Grimshaw JM, et al. The relationship between organisational characteristics and the effects of clinical guidelines on medical performance in hospitals, a meta-analysis. BMC Health Serv Res. 2006;6(1):53. http://doi.org/10.1186/1472-6963-6-53

27. Brusamento S, Legido-Quigley H, Panteli D, Turk E, Knai C, Saliba $\mathrm{V}$, et al. Assessing the effectiveness of strategies to implement clinical guidelines for the management of chronic diseases at primary care level in EU Member States: A systematic review. Health Policy. 2012;107(2-3):168-83. https://doi.org/10.1016/j. healthpol.2012.08.005

28. Allanson ER, Tunçalp Ö, Vogel JP, Khan DN, Oladapo OT, Long $\mathrm{Q}$, et al. Implementation of effective practices in health facilities: a systematic review of cluster randomised trials. BMJ Glob Heal. 2017;2(2):e000266. https://doi.org/10.1136/bmjgh-2016-000266

29. Brennan N, Mattick K. A systematic review of educational interventions to change behaviour of prescribers in hospital settings, with a particular emphasis on new prescribers. Br J Clin Pharmacol. 2013;75(2):359-72. http://doi.org/10.1111/j.1365-2125.2012.04397.x

30. Goodwin V, Jones-Hughes T, Thompson-Coon J, Boddy K, Stein $\mathrm{K}$. Implementing the evidence for preventing falls among community-dwelling older people: a systematic review. J Safety Res. 2011;42(6):443-51. https://doi.org/10.1016/j.jsr.2011.07.008

31. Häggman-Laitila A, Mattila L-R, Melender H-L. A systematic review of the outcomes of educational interventions relevant to nurses with simultaneous strategies for guideline implementation. J Clin Nurs. 2017;26(3-4):320-40. http://doi.org/10.1111/jocn.13405

32. Tooher R, Middleton P, Babidge W. Implementation of pressure ulcer guidelines: what constitutes a successful strategy? J Wound Care. 2003;12(10):373-82. https://doi/10.12968/jowc.2003.12.10.26551

33. Thomas LH, McColl E, Cullum N, Rousseau N, Soutter J, Steen N. Effect of clinical guidelines in nursing, midwifery, and the therapies: a systematic review of evaluations. Qual Health Care. 1998;7(4):18391. http://doi.org/10.1136/qshc.7.4.183

34. Gross PA, Pujat D. Implementing practice guidelines for appropriate antimicrobial usage: a systematic review. Med Care. 2001;39(8, Supp II):II-55-II-69.

35. Thomas LH, McColl E, Cullum N, Rousseau N, Soutter J. Clinical guidelines in nursing, midwifery and the therapies: a systematic review. J Adv Nurs. 1999;30(1):40-50. https://doi. org/10.1046/j.1365-2648.1999.01047.x

36. Wensing $M$, van der Weijden T, Grol R. Implementing guidelines and innovations in general practice: which interventions are effective? Br J Gen Pract. 1998;48(427):991-7.

37. Medves J, Godfrey C, Turner C, Paterson M, Harrison M, MacKenzie L, et al. Systematic review of practice guideline dissemination and implementation strategies for healthcare teams and team-based practice. Int J Evid Based Healthc. 2010;8(2):79-89. http://doi. org/10.1111/j.1744-1609.2010.00166.x

38. Fønhus MS, Dalsbø TK, Johansen M, Fretheim A, Skirbekk H, Flottorp SA. Patient-mediated interventions to improve professional practice. Cochrane Database Syst Rev. 2018;9:CD012472. http:/ /doi. org/10.1002/14651858.CD012472.pub2

39. O'Brien MA, Rogers S, Jamtvedt G, Oxman AD, Odgaard-Jensen J, Kristoffersen DT, et al. Educational outreach visits: effects on professional practice and health care outcomes. Cochrane Database Syst Rev. 2007;4:CD000409. http://doi.org/10.1002/14651858. CD000409.pub2

40. Ivers N, Jamtvedt G, Flottorp S, Young JM, Odgaard-Jensen J, French $\mathrm{SD}$, et al. Audit and feedback: effects on professional practice and healthcare outcomes. Cochrane Database Syst Rev. 2012;6:CD000259. http://doi.org/10.1002/14651858.CD000259.pub3

41. Stokes T, Shaw EJ, Camosso-Stefinovic J, Imamura M, Kanguru L, Hussein J. Barriers and enablers to guideline implementation strategies to improve obstetric care practice in low- and middle-income countries: a systematic review of qualitative evidence. Implement Sci. 2016;11(1):144. http://doi.org/10.1186/s13012-016-0508-1

42. Chakkalakal RJ, Cherlin E, Thompson J, Lindfield T, Lawson R, Bradley EH. Implementing clinical guidelines in low-income settings: A review of literature. Glob Public Health. 2013;8(7):784-95. http:/ / doi.org/10.1080/17441692.2013.81579

43. Solberg LI. Guideline implementation: what the literature doesn't tell us. Jt Comm J Qual Improv. 2000;26(9):525-37. https://doi. org/10.1016/S1070-3241(00)26044-6

44. Arditi C, Rège-Walther M, Durieux P, Burnand B. Computer-generated reminders delivered on paper to healthcare professionals: effects on professional practice and healthcare outcomes. Cochrane Database Syst Rev. 2017;7:CD001175. http://doi.org/10.1002/14651858. CD001175.pub4

45. Dexheimer JW, Borycki EM, Chiu K-W, Johnson KB, Aronsky D. A systematic review of the implementation and impact of asthma 
protocols. BMC Med Inform Decis Mak. 2014;14(1):82. https://doi. org /10.1186/1472-6947-14-82

46. May CR, Sibley A, Hunt K. The nursing work of hospital-based clinical practice guideline implementation: an explanatory systematic review using Normalisation Process Theory. Int J Nurs Stud. 2014;51(2):289-99. https:/ / doi.org/10.1016/j.ijnurstu.2013.06.019

47. Nguyen et al. How does integrated knowledge translation (IKT) compare to other collaborative research approaches to generating and translating knowledge? Learning from experts in the field. Health Research Policy and Systems. 2020; 18:35.

48. Biermann O, Kuchenmüller T, Panisset U, Leys M. Policy dialogues: facilitators' perceived role and influence. Int J Health Governance. 2018; 23(2): 120-33. https:/ / doi.org/10.1108/IJHG-12-2017-0063

49. Moat KA, Lavis JN, Clancy SJ, El-Jardali F, Pantoja T; Knowledge Translation Platform Evaluation study team. Evidence briefs and deliberative dialogues: perceptions and intentions to act on what was learnt. Bull World Health Organ. 2014;92(1):20-8. https://doi. org/10.2471/BLT.12.116806
50. Boyko JA, Lavis JN, Abelson J, Dobbins M, Carter N. Deliberative dialogues as a mechanism for knowledge translation and exchange in health systems decision-making. Soc Sci Med. 2012;75(11):193845. https://doi.org/10.1016/j.socscimed.2012.06.016

51. Morgan RL, Kelley L, Guyatt GH, Johnson A, Lavis JN. Decision-making frameworks and considerations for informing coverage decisions for healthcare interventions: a critical interpretive synthesis. J Clin Epidemiol. 2018;94:143-50. https://doi.org/10.1016/j. jclinepi.2017.09.023

Manuscrito recebido em 29 de junho de 2020. Aceito em versão revisada em 3 de setembro de 2020

\section{Implementation of the National Childbirth Guidelines in Brazil: barriers and strategies}

\section{ABSTRACT}

Keywords
The present report describes the process and results obtained with a knowledge translation project developed in three stages to identify barriers to the National Childbirth Guidelines in Brazil as well strategies for effective implementation. The Improving Programme Implementation through Embedded Research (iPIER) model and the Supporting Policy Relevant Reviews and Trials (SUPPORT) tools provided the methodological framework for the project. In the first stage, the quality of the Guidelines was evaluated and the barriers preventing implementation of the recommendations were identified through review of the global evidence and analysis of contributions obtained in a public consultation process. In the second stage, an evidence synthesis was used as basis for a deliberative dialogue aimed at prioritizing the barriers identified. Finally, a second evidence synthesis was presented in a new deliberative dialogue to discuss six options to address the prioritized barriers: 1) promote the use of multifaceted interventions; 2) promote educational interventions for the adoption of guidelines; 3) perform audits and provide feedback to adjust professional practice; 4) use reminders to mediate the interaction between workers and service users; 5) enable patient-mediated interventions; and 6) engage opinion leaders to promote the use of guidelines. The processes and results associated with each stage were documented and formulated to inform a review and update of the Guidelines and the development of an implementation plan for the recommendations. An effective implementation of the Guidelines is relevant to improve the care provided during labor and childbirth in Brazil.

Evidence-informed policy; implementation science; Evidence-based practice; practice guideline; natural childbirth; Brazil. 


\section{Obstáculos y estrategias para la aplicación de las Directrices Nacionales para el Parto Normal en el Brasil}

RESUMEN En este informe se presentan los procesos y resultados de un proyecto de traducción de conocimiento desarrollado en tres etapas para identificar los obstáculos y las estrategias para la aplicación efectiva de las Directrices Nacionales para el Parto Normal en el Brasil. El marco metodológico adoptado comprendió la iniciativa iPIER (Improving Program Implementation through Embedded Research) y las herramientas SUPPORT para políticas basadas en evidencia. En la primera etapa se evaluó la calidad de las Directrices y se identificaron los obstáculos a la aplicación de las recomendaciones, teniendo en cuenta la evidencia mundial y el análisis de las contribuciones obtenidas mediante una consulta pública. En la segunda etapa, una síntesis de la evidencia sirvió de base para un diálogo deliberativo para la priorización de los obstáculos. Por último, una nueva síntesis de la evidencia sirvió de base para un segundo diálogo deliberativo y presentó seis opciones para hacer frente a los obstáculos priorizados: 1) fomentar el uso de estrategias de intervención polifacéticas; 2) promover intervenciones educativas para mejorar el uso de directrices sanitarias; 3) realizar auditorías y proporcionar retroalimentación para la adecuación de la práctica profesional; 4) utilizar recordatorios para mediar en la interacción entre profesionales y usuarios; 5) permitir intervenciones mediadas por el paciente; y 6) incluir a líderes de opinión para fomentar el uso de directrices sanitarias. Se documentaron y presentaron los procesos y resultados de cada etapa del proyecto para fundamentar la actualización de las Directrices y la elaboración de un plan de aplicación de las recomendaciones. La aplicación efectiva de las Directrices es importante para mejorar la atención del parto y el nacimiento en el Brasil.

Keywords Política informada por la evidencia; ciencia de la implementación; práctica clínica basada en la evidencia; guía de práctica clínica; parto normal; Brasil. 